\title{
Molecular forms of butyrylcholinesterase and obesity
}

\author{
Dellyana R. Boberg, Lupe Furtado-Alle, Ricardo L.R. Souza and Eleidi A. Chautard-Freire-Maia \\ Department of Genetics, Federal University of Paraná, Curitiba, PR, Brazil.
}

\begin{abstract}
This study compared obese $(\mathrm{N}=134)$ and unobese $(\mathrm{N}=92)$ male blood donors, regarding the relative intensity (RI) and activity of different molecular forms (G1, G2, G4 and G1-ALB) of butyrylcholinesterase (BChE, EC 3.1.1.8) found in plasma, thereby searching for an association between these variables with obesity and SNPs of exons 1 and 4 of the $B C H E$ gene. It was shown that obese and unobese individuals do not differ in the RI of each $B C h E$ band, even when classifying the sample into three genotypes of exons 1 and 4 of the BCHE gene (-116GG/539AA, $-116 G G / 539 A T,-116 G A / 539 A T)$. Although the mean BChE activity of each band was significantly higher in obese than in unobese blood donors, the proportions of BChE bands were maintained, even under the metabolic stress associated to obesity, thereby leading to infer that this proportion is somehow regulated, and may therefore be important for BChE functions.
\end{abstract}

Key words: butyrylcholinesterase, body mass index, obesity, $B C H E$ gene SNPs.

Received: October 20, 2009; Accepted: March 30, 2010.

Human butyrylcholinesterase (BChE; EC 3.1.1.8) hydrolyses choline and other esters, this synthesis taking place in the liver, with posterior distribution to several parts of the organism. $\mathrm{BChE}$ is coded by the BCHE gene (3q26.1-q26.2) which presents four exons (Arpagaus et al., 1990), with more than 70 already-described variants (review in Souza et al., 2005b). Another type of genetic variation occurs through the interaction of products from the $B C H E$ and CHE2 (2q33-q35) genes, this resulting in the CHE2 C5+ and CHE2 C5- phenotypes, characterized by the presence and absence of the $\mathrm{C}_{5}$ complex, respectively (Harris et al., 1962).

$\mathrm{BChE}$ is found in human plasma, either in homomeric, viz., monomers (G1), dimers (G2), trimers (G3) and tetramers (G4) (Harris et al., 1962), or heteromeric forms associated with other substances, such as albumin (G1ALB) (Masson, 1989).

Although the physiological role of $\mathrm{BChE}$ has not been fully elucidated, published data suggest its relationship with lipid metabolism (Kutty et al., 1977), weight (Chautard-Freire-Maia et al., 1991; Li et al., 2008) and body mass index (BMI) (Alcântara et al., 2001, 2003; Souza et al., 2005a; Furtado-Alle et al., 2008). Besides mean $\mathrm{BChE}$ activity tending to be higher in obese than in unobese individuals (Chautard-Freire-Maia et al., 1991; Alcântara et al., 2003; Furtado-Alle et al., 2008), BChE itself is also related to ghrelin (Kojima et al., 2001), a peptide associated with obesity (Tschöp et al., 2001), through its

Send correspondence to Eleidi A. Chautard-Freire-Maia. Caixa Postal 19071, 81531-990 Curitiba, PR, Brazil. E-mail: eleidi@ ufpr.br. hydrolyzing and inactivating the latter (De Vriese et al., 2004).

The aim was to compare the relative intensity (RI) and activity of BChE bands (G1, G1-ALB, G2 and G4) in obese and control individuals, and search for an association between the activity of each band with obesity and SNPs of exons 1 and 4 of the $B C H E$ gene. This research was approved by the Ethical Committee (CONEP; registration number 2063).

The sample comprised 134 obese (BMI $\geq 30$; mean age 36.9) and 92 unobese $(20 \leq \mathrm{BMI}<25$; mean age 35.1$)$ male blood donors bearing the CHE2 C5- phenotype. Individuals with any no detectable $\mathrm{BChE}$ band were excluded from the study. The detection of $\mathrm{BChE}$ bands in plasma stored from eight to eleven years at $-20^{\circ} \mathrm{C}$ - was by means of electrophoresis $\left(8 \mathrm{~h} ; 100 \mathrm{~V}\right.$ and $\left.11 \mathrm{~mA} ; 4^{\circ} \mathrm{C}\right)$ of plasma samples $(5 \mu \mathrm{L})$ mixed with $7 \mu \mathrm{L}$ of bromophenol blue before loading onto polyacrylamide gels $(170 \times 160 \times$ $0.8 \mathrm{~mm} ; 29: 1$ stock solution) of $5 \%$ (1 cm; stacking gel) and $7 \%$ (13 cm; resolving gel) concentrations in TBE $1 \mathrm{X}$. The bands were revealed at $37^{\circ} \mathrm{C}$ after 30 to $40 \mathrm{~min}$ incubation in a solution of $0.2 \%$ Fast Red TR in $200 \mathrm{mM}$ sodium phosphate buffer $(\mathrm{pH} 7.1)$ containing $3.4 \%$ of $30 \mathrm{mM}$ alphanaphthyl acetate dissolved in acetone, the volume being completed with distilled water (1:1). A pre-run of $30 \mathrm{~min}$ was done before plasma loading. Data concerning plasma BChE activity (Dietz et al., 1972), as modified (Evans and Wroe, 1978), and genotypes of exons $1(-116 G G,-116 G A$, $-116 A A)$ and 4 (539AA, 539AT, 539TT) of the BCHE gene were obtained from the data bank of the Laboratory of Polymorphisms and Linkage (Genetics Department, 
Table 1 - Means \pm S. D. of relative intensity and activity of butyrylcholinesterase $(\mathrm{BChE})$ bands in obese $(\mathrm{N}=134)$ and unobese $(\mathrm{N}=92)$ male blood donors, showing the results of t-test comparisons.

\begin{tabular}{|c|c|c|c|c|c|c|c|}
\hline \multirow[t]{3}{*}{ BChE bands } & \multicolumn{3}{|c|}{ Relative intensity } & \multicolumn{4}{|c|}{ Activity (KU/L) } \\
\hline & \multicolumn{2}{|c|}{ Means \pm S.D. } & \multirow[t]{2}{*}{ t-test (p) } & \multicolumn{2}{|c|}{ Means \pm S.D. } & \multirow[t]{2}{*}{ t-test (p) } & \multirow{2}{*}{$\begin{array}{c}\text { Means ratio } \\
(\mathrm{O} / \mathrm{UO})\end{array}$} \\
\hline & $\mathrm{O}$ & UO & & $\mathrm{O}$ & UO & & \\
\hline G4 & $0.68 \pm 0.13$ & $0.70 \pm 0.12$ & $0.96(>0.30)$ & $4.39 \pm 1.98$ & $3.20 \pm 0.86$ & $6.18\left(<10^{-8}\right)$ & 1.37 \\
\hline G2 & $0.04 \pm 0.03$ & $0.04 \pm 0.03$ & $0.11(>0.90)$ & $0.27 \pm 0.23$ & $0.19 \pm 0.14$ & $3.37\left(<10^{-3}\right)$ & 1.42 \\
\hline G1-ALB & $0.09 \pm 0.06$ & $0.09 \pm 0.06$ & $0.79(>0.40)$ & $0.58 \pm 0.43$ & $0.44 \pm 0.34$ & $2.75\left(<10^{-2}\right)$ & 1.32 \\
\hline G1 & $0.19 \pm 0.10$ & $0.17 \pm 0.09$ & $1.72(>0.05)$ & $1.27 \pm 1.06$ & $0.78 \pm 0.50$ & $4.65\left(<10^{-5}\right)$ & 1.63 \\
\hline
\end{tabular}

S.D. $=$ Standard Deviation, $\mathrm{O}=$ Obese sample, $\mathrm{UO}=$ Unobese sample.

UFPR), most of which part of a previous study (FurtadoAlle et al., 2008). The activity of each band was a result of the multiplication of plasma BChE activity by the RI of each $\mathrm{BChE}$ band detected in the polyacrylamide gel, subsequently measured by optical densitometry with $K O D A K$ 1D Image Analysis Software. Statistica for Windows (StatSoft, Inc., 5.5 version, 2000) was used for statistical analysis.

Mean $\mathrm{BChE}$ activity in plasma, as estimated for the present sample, was significantly higher in obese $(6.51 \pm 2.87)$ than in unobese men $(4.61 \pm 1.11 ; \mathrm{t}=6,949$, $\left.\mathrm{p}=6 \times 10^{-11}\right)$. Table 1 shows that obese and control individuals do not differ in the proportions of each $\mathrm{BChE}$ band, although the activity of each band is significantly higher in obese than in unobese men, with ratios varying from 1.32 (G1-ALB) to 1.63 (G1). When classified according to the more frequent genotypes, mean $\mathrm{BChE}$ activity in plasma is significantly higher in obese than unobese individuals, respectively: $-116 G G / 539 A A(\mathrm{~N}=80,6.58 \pm 2.66$ and $\left.\mathrm{N}=54,5.02 \pm 1.10 ; \mathrm{t}=4.67, \mathrm{p}<10^{-5}\right),-116 G G / 539 A T$ $(\mathrm{N}=20,7.08 \pm 3.91$ and $\mathrm{N}=12,3.96 \pm 1.05 ; \mathrm{t}=3.37$, $\mathrm{p}<0.005)$ and $-116 G A / 539 A T(\mathrm{~N}=19,5.22 \pm 2.22$ and $\mathrm{N}=12,3.95 \pm 0.78 ; \mathrm{t}=2.26, \mathrm{p}<0.05)$. As to RI means of $\mathrm{BChE}$ bands, classified according to these genotypes, no significant difference between obese and control individuals was revealed through t-tests (data not shown).

The $K$ mutation (539T) of the BCHE gene was associated with lower BChE activity (Rubinstein et al., 1978), and it was shown that the -116A SNP is preferentially found in cis combination with the $539 T$ variant (Bartels et al., 1990). Recently, it was reported that the 539T SNP alone is not associated with decreased $\mathrm{BChE}$ activity, since this requires the 5' UTR -116A variant, probably through the latter affecting transcription and/or translation of the $B C H E$ gene (Furtado-Alle et al., 2008). From the present data, it can be seen that obese and control samples, grouped by genotypes of exons 1 and 4 of the $B C H E$ gene, revealed similar RI of each $\mathrm{BChE}$ band. Therefore, the reduced plasma $\mathrm{BChE}$ activity associated with genotype -116GA/539AT (Furtado-Alle et al., 2008) does not seem to affect the RI of the $\mathrm{BChE}$ molecular forms examined.
On considering the encountered similarity of RI in each $\mathrm{BChE}$ band in obese and control subjects, the higher mean activity of each band in the former can be attributed solely to higher BChE plasma activity. Even though this activity is higher in obese individuals and lower in the $-116 G A / 539 A T$ genotype than in others, from the data, it can be seen that the RI of each band is maintained, independently of obesity and the examined genotypes, suggesting that this proportion is regulated, and may therefore be important for BChE function(s).

\section{Acknowledgments}

Grants were received from CNPq, Fundação Araucária and CAPES.

\section{References}

Alcântara VM, Rodrigues LC, Oliveira LC and Chautard-FreireMaia EA (2001) Association of the CHE2 locus with body mass index and butyrylcholinesterase activity. Hum Biol 73:587-595.

Alcântara VM, Oliveira LC, Réa RR, Suplicy HL and ChautardFreire-Maia EA (2003) Butyrylcholinesterase and obesity in individuals with the CHE2 C5+ and CHE2 C5- phenotypes. Int J Obes 27:1557-1564.

Arpagaus M, Kott M, Vatsis KP, Bartels CF, La Du BN and Lockridge O (1990) Structure of the gene for human butyrylcholinesterase. Evidence for a single copy. Biochemistry 29:24-131.

Bartels CF, van der Spek AFL and La Du BN (1990) Two polymorphisms in the non-coding regions of the $B C H E$ gene. Nucleic Acids Res 18:6171.

Chautard-Freire-Maia EA, Primo-Parmo SL, Picheth G, Lourenço MAC and Vieira MM (1991) The $\mathrm{C}_{5}$ isozyme of serum cholinesterase and adult weight. Hum Hered 41:330339.

De Vriese C, Gregoire F, Lema-Kisoka R, Waelbroeck M, Robberecht P and Delporte C (2004) Ghrelin degradation by serum and tissue homogenates: Identification of the cleavage sites. Endocrinology 145:4997-5005.

Dietz AA, Rubinstein HM, Lubrano TE and Hodges LK (1972) Improved method for the differentiation of cholinesterase variants. Am J Hum Genet 24:58-64. 
Evans RT and Wroe J (1978) Is serum cholinesterase activity a predictor of succinyl choline sensitivity? An assessment of four methods. Clin Chem 24:1762-1766.

Furtado-Alle L, Andrade FA, Nunes K, Mikami LR, Souza RLR and Chautard-Freire-Maia EA (2008) Association of variants of the -116 site of the butyrylcholinesterase $B C H E$ gene to enzyme activity and body mass index. Chem Biol Interact 175:115-118.

Harris H, Hopkinson DA and Robson EB (1962) Two-dimensional electrophoresis of pseudocholinesterase components in normal human serum. Nature 196:1296-1298.

Kojima M, Hosoda H, Matsuo H and Kangawa K (2001) Ghrelin: Discovery of the natural endogenous ligand for the growth hormone secretagogue receptor. Trends Endocrinol Metab 12:118-122.

Kutty KM, Redheendran R and Murphy D (1977) Serum cholinesterase: Funcion in lipoprotein metabolism. Experientia 33:420-421.

Li B, Duysen EG and Lockridge O (2008) The butyrylcholinesterase knockout mouse is obese on a high-fat diet. Chem Biol Interact 175:88-91.
Masson P (1989) A naturally occurring molecular form of human plasma cholinesterase is an albumin conjugate. Biochim Biophys Acta 988:258-266.

Rubinstein HM, Dietz AA and Lubrano T (1978) $\mathrm{E} 1^{\mathrm{K}}$, another quantitative variant at cholinesterase locus 1 . J Med Genet 15:27-29.

Souza RLR, Fadel-Picheth C, Allebrandt KV, Furtado L and Chautard-Freire-Maia EA (2005a) Possible influence of BCHE locus of butyrylcholinesterase on stature and body mass index. Am J Phys Anthropol 126:329-334.

Souza RLR, Mikami LR, Maegawa ROB and Chautard-FreireMaia EA (2005b) Four new mutations in the BCHE gene of butyrylcholinesterase in a Brazilian blood donor sample. Mol Genet Metab 84:349-353.

Tschöp M, Weyer C, Tataranni PA, Devanarayan V, Ravussin E and Heiman ML (2001) Circulating ghrelin levels are decreased in human obesity. Diabetes 50:707-709.

Associate Editor: Francisco Mauro Salzano

License information: This is an open-access article distributed under the terms of the Creative Commons Attribution License, which permits unrestricted use, distribution, and reproduction in any medium, provided the original work is properly cited. 